Abstract AB0622 - Table 1. Clinical characteristics of the cohort

\begin{tabular}{lc}
\hline Characteristics & Values \\
\hline Age, mean (S.D.) years & $46.9(13.9)$ \\
Caucasian race, $\mathbf{n} / \mathbf{N}(\%)$ & $90 / 92(97.8)$ \\
Disease duration, median (range) years & $13(0-35)$ \\
Age of SLE onset, mean (S.D.) years & $33.8(12.8)$ \\
BMI, median (range) $\mathbf{k g} / \mathbf{m}^{2}$ & $26.5(17.7-53.5)$ \\
Current smoker, $\mathbf{n} / \mathbf{N}(\%)$ & $14 / 92(15.2)$ \\
Family history of SLE in first degree relative, $\mathbf{n} / \mathbf{N}(\%)$ & $3 / 92(3.3)$ \\
Osteopaenia/osteoporosis, $\mathbf{n} / \mathbf{N}(\%)$ & $30 / 92(32.6)$ \\
Hypertension, $\mathbf{n} / \mathbf{N}(\%)$ & $22 / 92(23.9)$ \\
Diabetes mellitus, $\mathbf{n} / \mathbf{N}(\%)$ & $7 / 92(7.6)$ \\
Fibromyalgia, $\mathbf{n} / \mathbf{N}(\%)$ & $9 / 92(9.8)$ \\
Anti-phospholipid syndrome, $\mathbf{n} / \mathbf{N}(\%)$ & $7 / 92(7.6)$ \\
Sjogren's syndrome, $\mathbf{n} / \mathbf{N}(\%)$ & $4 / 92(4.3)$ \\
Rheumatoid arthritis, $\mathbf{n} / \mathbf{N}(\%)$ & $3 / 92(3.3)$ \\
Current prednisolone, $\mathbf{n} / \mathbf{N}(\%)$ & $41 / 92(44.6)$ \\
Current hydroxychloroquine, $\mathbf{n} / \mathbf{N}(\%)$ & $55 / 92(59.8)$ \\
Current azathioprine, $\mathbf{n} / \mathbf{N}(\%)$ & $20 / 92(21.7)$ \\
Current methotrexate, $\mathbf{n} / \mathbf{N}(\%)$ & $10 / 92(10.9)$ \\
Current mycophenolate, $\mathbf{n} / \mathbf{N}(\%)$ & $6 / 92(6.5)$ \\
\hline
\end{tabular}

Conclusions: This is the first population based study on SLE to be carried out in Malta. The prevalence of SLE in Malta is estimated to be 25.5 patients per 100000 and the estimated incidence is 1.05 patients per 100000 per year. A high frequency of obesity and vitamin $D$ deficiency and insufficiency were noted in SLE patients. Other unmet needs include an uncontrolled disease activity, fatigue, poor sleep quality and anxiety.

Disclosure of Interest: None declared

DOI: 10.1136/annrheumdis-2018-eular.1722

\section{AB0623 PREGNANCY OUTCOME IN SYSTEMIC LUPUS ERYTHEMATOSUS: A RETORSPECTIVE STUDY}

R. Dumitriu ${ }^{1}$, C. Vasiliu' ${ }^{2}{ }^{1}$ Infertility and gonadal pathology, National Institute of Endocrinology ,,C.I. Parhon”; ${ }^{2}$ Obstetrics and Gynecology, Bucharest Emergency University Hospital, Bucharest, Romania

Background: Pregnancy represents a challenge for patients with systemic lupus erythematosus. One of the major risk is the occurence of a flare during pregnancy. The influences are mutual, and the risk of complications depend mostly on the dis ease activity in the last 6-12 months before pregnancy. Therefore, these patients need a multidisciplinary approach, the obstetrician should collaborate with the rheumatologist and nephorologist.

Objectives: To determinate the associations between disease activity and pregnancy outcomes, and the risk factors that predict pregnancy complications and flare.

Methods: We present a retrospective study conducted between january 2010 and december 2015. We enrolled 35 pregnant patients, diagnosed with SLE with ages between 21 and 46 years old. All patients were followed up since the beginning of the pregnancy until delivery. We tested the correlations between different biomarkers and clinical manifestations of disease activity and pregnancy outcomes.

Results: Maternal complications occured in $71,24 \%$ of the cases. The most common complications were: miscarriages, hematologic abnormalities (anemias, thrombocytopenia), premature birth and preeclampsia. The prematurity rate was $11 \%$ and most common delivery mode was the caesarian section. Also, the planning of the pregnancy was a predictor of a good fetal outcome $(p=0,01)$. The presence of the lupus anticoagulant was associated with prematurity $(p=0,046)$ at univaryate analysis. Antiphospholipidic syndrome was associated in $12 \%$ of the cases. Univaryate analysis did not show a correlation between the presence of the syndrome and any pregnancy outcome.

Abstract AB0623 - Table 1. Risk Estimate preeclampsia in patients with APS

\begin{tabular}{llll}
\hline & Value & \multicolumn{2}{l}{ 95\% Confidence Interval } \\
\hline & & Lower & Upper \\
\hline Odds Ratio for Preeclampsia & 6667 &, 607 & 73195 \\
For cohort APS=yes & 2417 & 1104 & 5292 \\
For cohort APS=no &, 363 &, 065 & 2014 \\
N of Valid Cases & 33 & & \\
\hline
\end{tabular}

Conclusions: Pregnancy should be planned when the disease is in remission. Lupus nephritis, is an important risk factor for preeclampsia. Also, the presence of lupus anticoagulant is a risk factor for preeclampsia, and hematologic determinations during pregnancy. The risk factors for pregnancy complications were; secondary antiphospholipid syndrome, presence of lupus nephropaty, thrombocytopenia. our study showes that the exacerbations depend on the disease activity in the moment of conception.

Disclosure of Interest: None declared

DOI: 10.1136/annrheumdis-2018-eular.1769

\section{AB0624 DYSLIPIDEMIA IN SYSTEMIC LUPUS ERYTHEMATOSUS: CORRELATION WITH DISEASE SEVERITY AND CYTOKINES}

S. Huang ${ }^{1}$, Z. Zhang ${ }^{1}$, on behalf of Department of Rheumatology and Immunology, The Affiliated Drum Tower Hospital of Nanjing University Medical School, S. Wu ${ }^{1}$, J. Qi ${ }^{1}$, D. Wang ${ }^{1}$, G. Yao ${ }^{1}$, L. Sun ${ }^{1}$

${ }^{1}$ Department of Rheumatology and Immunology, Drum Tower Clinical Medical College of Nanjing Medical University, Nanjing, China

Objectives: The patients with systemic lupus erythematosus (SLE) are obviously at high risk of cardiovascular disease (CVD) and the relationships between disturbed lipid metabolism and lupus activity remain to be elucidated. We evaluated dyslipidemia in association with disease severity, organ involvement and cytokines in patients with SLE.

Methods: Outpatients with SLE $(n=105)$ and healthy controls $(n=75)$ were recruited in this study. The concentrations of plasma tumour necrosis factor receptors A(sTNFRA), tumour necrosis factor receptors B(sTNFRB) and adipokine angiopoietin-like 4 (ANGPTL4)were measured by ELISA. The clinic and laboratory data were collected from patient records using electronic data processing The data collected included serum lipid (TG, TC, HDL, LDL, ApoA1, ApoB), renal function (proteinuria, albuminuria, creatinine, blood urea nitrogen, uric acid), liver function (Alanine transaminase [ALT], glutamic oxalacetic transaminase [AST], total protein, albumin, globulin), blood system (lymphocyte, white blood cell [WBC], platelet[PLT], haemoglobin[HB]).

Results: Compared with the healthy controls, the level of serum TG, TC, LDL, $A p o B$ were significantly increased, while $H D L$ and $A p o A 1$ were decreased. The serum levels of LDL and ApoB were positively correlated to SLEDAI, while HDL and ApoA1 were negatively correlated to SLEDAI. The patients with lupus nephritis had more severe dyslipidemia. The blood TG, TC, LDL, ApoB levels were positively correlated to 24 hour proteinuria and serum creatinine, urea nitrogen, uric acid. The blood levels of TG, TC, LDL, ApoB were positively correlated to serum total protein, albumin, globulin. The serum level of ANGPTL4 was positively correlated to HDL and ApoA1 as well as sTNFRA and STNFRB with TG

Conclusions: Dyslipidemia in SLE was significantly correlated with SLEDAI and kidney involvement. The circulated levels of sTNFRA, sTNFRB and ANGPTL4 were associated with dyslipidemia in SLE.

Disclosure of Interest: None declared

DOI: 10.1136/annrheumdis-2018-eular.4054

\section{AB0625 COHORT OF PATIENTS: A MULTICENTER STUDY}

S.A. Elbakry ${ }^{1}$, N. Afifi ${ }^{1}$, S.A. Hussein ${ }^{1}$, N. Mohannad ${ }^{2}$, I.H. Bassyouni ${ }^{3}$, N.F. Abou elezz ${ }^{4} .{ }^{1}$ Department of internal Medicine-Rheumatology Division, Ain Shams University-Cairo-Egypt, Cairo; ${ }^{2}$ Department of internal Medicine-Rheumatology Division, Alexandria University Hospitals-Alexandria, Alexandria; ${ }^{3}$ Rheumatology and Rehabilitation Department, Faculty of Medicine, Cairo University, ${ }^{4}$ Community and Public Health Department Faculty of Medicine, Ain Shams University-CairoEgypt, Cairo, Egypt

Background: Systemic lupus erythematosus (SLE) is a chronic systemic autoim mune disease that mainly affects females in the reproductive age. ${ }^{1}$ The disease presents with a diverse spectrum of clinical and immunological manifestations which has been studied in many countries and ethnic groups. Data from North Africa especially Egypt are minimal.

Objectives: To study the clinical and immunological characteristics of an Egyptian cohort with SLE and compare it with data from MENA region and international data.

Methods: In this retrospective study, data of 569 SLE patients who fulfilled the modified American College of Rheumatology (ACR) criteria for the diagnosis of $\mathrm{SLE}^{2}$ were collected from three tertiary care centres in Cairo and Alexandria from the period of January 2014 to December 2017. Disease activity was assessed by using the SLE disease activity index (SLEDAI). ${ }^{3}$

Results: Of 569 patients $92.6 \%$ were females and $4.7 \%$ males with mean age at presentation $26.3 \pm 8.8$ years and median disease duration four years ( $\mathrm{min} 0.08$ max 30 years). The main presenting symptom was musculoskeletal (arthritis/ arthralgia) in $44.1 \%$ followed by fever in $39.4 \%$ and nephritis in $14.2 \%$. Renal affection was present in 374 patients (65.7\%) and renal biopsy was done in 268 patients with the most common is class III and IV lupus nephritis $\{18.3 \%$ and $14.1 \%$ respectively\} (table 1 ). Antinuclear antibodies (ANA) was positive in all patients and immunofluorescence pattern was done in 256 patients; homogenous 